\title{
DESIGN AND OPTIMIZATION OF PEDIATRIC CEFUROXIME AXETIL DISPERSIBLE TABLET CONTAINING ION-EXCHANGE RESIN
}

\author{
NISHANT OZA*, SWATI SAGAR \\ C. U. Shah College of Pharmacy and Research, Wadhwan City 363030, Gujarat, India \\ Email: ozanishant@gmail.com \\ Received: 12 Jan 2019, Revised and Accepted: 24 May 2019
}

\begin{abstract}
Objective: The aim of present work was to develop of pediatric cefuroxime axetil $125 \mathrm{mg}$ dispersible tablets by using ion exchange resin as a taste masking agent and quality target product profile was defined based on the properties of the cefuroxime axetil.

Methods: Initially, cefuroxime axetil and various resin complexes (DRC) were prepared with different conditions and evaluated for taste masking and drug loading. Optimized DRC was used to formulate the dispersible tablet. A $3^{2}$ full factorial design was employed to study the effect of mannitol $\left(\mathrm{X}_{1}\right)$ and microcrystalline cellulose PH-101 $\left(\mathrm{X}_{2}\right)$ on drug release at $10 \mathrm{~min}$ and time taken to $80 \%$ drug release. In the present study, the following constraints were arbitrarily used for the selection of an optimized batch: $\mathrm{Q}_{10}>65 \%$ and $\mathrm{T}_{80 \%<}<30$ min. Multiple linear regression analysis, ANOVA and graphical representation of the influence factor by 3D plots were performed by using Sigmaplot 11.0. Checkpoint batch was prepared to validate the evolved model.
\end{abstract}

Results: Among the various drug resins complex DRC-9 was found with less bitter taste which was containing kyron T-114 and among the all factorial batch $\mathrm{F}_{7}$ showed highest drug release at $10 \mathrm{~min}\left(\mathrm{Q}_{10}\right)$ and lowest time taken to $80 \%$ drug release $\left(\mathrm{T}_{80}\right)$ hence batch $\mathrm{F}_{7}$ was selected as an optimized batch and it's found to be stable in the stability evaluation.

Conclusion: The results of full factorial design indicate mannitol and MCC PH-101 have a significant effect on drug release.

Keywords: Taste masking, Ion exchange resin, Cefuroxime axetil, Dispersible Tablet, 32Full Factorial Design

(C) 2019 The Authors. Published by Innovare Academic Sciences Pvt Ltd. This is an open access article under the CC BY license (http://creativecommons.org/licenses/by/4.0/) DOI: http://dx.doi.org/10.22159/ijap.2019v11i4.32012

\section{INTRODUCTION}

Oral route of drug administration is the most appealing route for drug delivery. Among the various dosage forms tablet is one of the most preferred dosage forms because of its ease of manufacturing, convenience in administration, accurate dosing, and stability compared with other forms etc. A number of orally administered drugs exhibit natural bitter taste that creates an unpleasant feeling in the mouth $[1,2]$. Therefore, it is necessary to reduce or mask the bitterness for enhancing patient acceptability and improving oral palatability of bitter drugs. Cefuroxime axetil has a broad spectrum antibacterial agent with a bitter taste, so it is necessary to mask the bitter taste for pediatric patients [3,4]. Different methods have been suggested for masking of the bitter taste, which includes coating inclusion complexes, molecular complexes, solid dispersion, microencapsulation, multiple emulsions, liposome's, Prodrugs and mass extrusion method from that ion exchange resin is one of most extensively method to overcome this problem $[5,6]$.

The present study was aimed to prepare the drug-resin complex (DRC) of cefuroxime axetil and evaluate for taste and drug loading. It was compressed into tablets by direct compression method. A $3^{2}$ full factorial design was employed to study the effect of mannitol $\left(\mathrm{X}_{1}\right)$ and MCC PH-101 $\left(\mathrm{X}_{2}\right)$ on drug release at $10 \mathrm{~min}\left(\mathrm{Q}_{10}\right)$ and time is taken to $80 \%$ drug release $\left(\mathrm{T}_{80}\right)$. In the present study, the following constraints were arbitrarily used for the selection of an optimized batch: $\mathrm{Q}_{10}>65 \%$ and $\mathrm{T}_{80 \%}<30 \mathrm{~min}[7,8]$.

\section{MATERIALS AND METHODS}

\section{Materials and reagents}

Cefuroxime axetil was received as a generous gift from Lincoln pharmaceutical Ltd., Ahmedabad, Gujarat, India. Kyron T 114 and kyron T 134 was obtained from Corel pharma ltd., Ahmedabad, Gujarat, India. Microcrystalline cellulose PH 101 and mannitol were purchased from Astron chemicals, Mumbai, Maharashtra, India. All other materials and chemicals used were of either pharmaceutical or analytical grade.

\section{Drug excipients compatibility study}

The physicochemical compatibilities of the drug and excipients were tested by differential scanning calorimetric (DSC) analysis. Thermograms of cefuroxime axetil, drug-resin complex and drugexcipients physical mixture were derived from DSC with a thermal analysis performed by using an automatic thermal analyzer system (DSC 60, Shimadzu, Japan). The analysis was performed at a rate of $20{ }^{\circ} \mathrm{C} / \mathrm{min}$ from $50{ }^{\circ} \mathrm{C}$ to $300{ }^{\circ} \mathrm{C}$ under a nitrogen flow of $25 \mathrm{ml} / \mathrm{min}$ $[9,10]$.

Development and evaluation of drug-resin complex (DRC)

In the batch process, the activated resin was placed in a beaker containing deionised water and allows swelling for $30 \mathrm{~min}$. accurately weighed cefuroxime axetil was added and stirred for different periods of time at various $\mathrm{pH}$ as shown in table 3 . The mixtures were filtered and the residue was washed with deionised water to remove the loosely adsorbed drug from the surface. DRC was allowed to dry at room temperature and was stored in a tightly closed container. DRC was evaluated for taste and drug loading. Taste of drug-resin complexes was subjected to gustatory sensory evaluation test performed by a panel of ten volunteers. The volunteers were selected randomly and instructed to rate the samples as per the taste evaluation scale. For drug loading analysis $50 \mathrm{mg}$ of DRC was added in 0.1 N HCL and kept in a sonicator for 30 min. The samples were withdrawn and taking absorbance at $277 \mathrm{~nm}$ $[11,12]$.

Preliminary trail of cefuroxime axetil $125 \mathrm{mg}$ dispersible tablets

Cefuroxime axetil $125 \mathrm{mg}$ dispersible tablets was prepared according to the formula given in table 1 . All the ingredients were passed through 60\# sieve separately and mixed in geometrical order. First MCC, mannitol, SSG were mixed together then add the DRC and mixed for $10 \mathrm{~min}$. Finally, sodium saccharin or aspartame, sodium stearyl fumarate or magnesium stearate and talc were mixed for $10 \mathrm{~min}$. Compression was carried out using $11 \mathrm{~mm}$ punch set. All the batches 
were stored properly and evaluation was carried out [13,14].

\section{Evaluation of cefuroxime axetil $125 \mathrm{mg}$ dispersible tablets}

Thickness, hardness, weight variation, \% friability and disintegration test of the formulations were measured as described by Yadav K et al., Khar RK et al., Madgulkar AR et al., and Lakade SH et al., respectively [15-18].

Wetting time: It can be measured by using five circular tissue papers of $10 \mathrm{~cm}$ in diameter, which are placed in a petridish of $10 \mathrm{~cm}$ diameter. $10 \mathrm{ml}$ of eosin solution is added to the petridish. A tablet is carefully placed on the surface of the tissue paper. The time required for water to reach the upper surface of the tablet is noted as the wetting time.

The fineness of dispersion: It is an assessment of the grittiness which arises due to the disintegration of the tablet into coarse particles. The test was performed by placing two tablets in $100 \mathrm{ml}$ water and stirring it gently, till the tablets get completely disintegrated. The formulation was considered to form a smooth dispersion if the complete dispersion passes through a sieve screen with a nominal mesh aperture of $710 \mu \mathrm{m}$ without leaving any residue on the mesh.

In vitro dissolution study: Drug release studies were carried out using by USP II dissolution test apparatus at $37 \pm 0.5^{\circ} \mathrm{C}$ and $50 \mathrm{rpm}$. In this study, $0.1 \mathrm{~N} \mathrm{HCl}(\mathrm{pH} \mathrm{1.2)}$ was used as a dissolution medium. Aliquots of $5 \mathrm{ml}$ were withdrawn at predetermined time intervals and an equal amount of fresh dissolution medium was added. The solution was suitably diluted and assayed at $277 \mathrm{~nm}$ using a UV-Vis double-beam spectrophotometer.

Taste analysis: All the batches of tablets were subjected to the gustatory sensory evaluation test performed by a panel of ten volunteers. The volunteers selected randomly and instructed to rate the samples as per the taste evaluation scale [19, 21].

Table 1: Preliminary trail formulation of cefuroxime axetil $125 \mathrm{mg}$ dispersible tablets

\begin{tabular}{|c|c|c|c|c|c|c|c|c|c|c|c|c|c|c|c|}
\hline S. No. & Ingredients & X1 & $\mathrm{X} 2$ & X3 & $\mathrm{X} 4$ & $\mathrm{X5}$ & X6 & $\mathbf{X 7}$ & X8 & X9 & $\mathrm{X} 10$ & $\mathrm{X11}$ & $\mathrm{X} 12$ & $\mathrm{X13}$ & $\mathrm{X} 14$ \\
\hline 1 & DRC* & 543 & 543 & 543 & 543 & 543 & 543 & 543 & 543 & 543 & 543 & 543 & 543 & 543 & 543 \\
\hline 2 & Crosspovidone & 20 & 25 & - & - & - & - & - & - & - & - & - & - & - & - \\
\hline 3 & Croscarmellose Sodium & - & - & 20 & 25 & - & - & 30 & - & 30 & - & - & - & - & - \\
\hline 4 & Sodium Starch Glycolate & - & - & - & - & 20 & 25 & - & 30 & - & 30 & 40 & 45 & 30 & 30 \\
\hline 5 & Aspartame & 20 & 20 & 20 & 20 & 20 & 20 & - & - & - & - & - & - & - & - \\
\hline 6 & Sodium Saccharin & - & - & - & - & - & - & 20 & 20 & 20 & 20 & 20 & 20 & 20 & 20 \\
\hline 7 & MCC PH-101 & 56 & 51 & 56 & 51 & 56 & 51 & 46 & 46 & - & - & - & - & 15 & 31 \\
\hline 8 & Mannitol & - & - & - & - & - & - & - & - & 46 & 46 & 46 & 46 & 31 & 15 \\
\hline 9 & Talc & 5 & 5 & 5 & 5 & 5 & 5 & 5 & 5 & 5 & 5 & 5 & 5 & 5 & 5 \\
\hline 10 & Magnesium Stearate & 7 & 7 & 7 & 7 & 7 & 7 & 7 & 7 & - & - & - & & - & - \\
\hline 11 & Sodium Stearyl Fumarate & - & - & - & - & - & - & - & - & 7 & 7 & 7 & 7 & 7 & 7 \\
\hline 12 & Flavour & 5 & 5 & 5 & 5 & 5 & 5 & 5 & 5 & 5 & 5 & 5 & 5 & 5 & 5 \\
\hline Total & & 656 & 656 & 656 & 656 & 656 & 656 & 656 & 656 & 656 & 656 & 666 & 671 & 660 & 660 \\
\hline
\end{tabular}

*Drug resin complex (DRC) contain 125 mg cefuroxime axetil and kyron T-114

\section{Optimization of excipients amount by using $3^{2}$ full factorial design}

A $3^{2}$ full factorial design was used in the present study. Formulation of factorial batches was shown in table 2. On the basis of preliminary results, the amount of mannitol $\left(\mathrm{X}_{1}\right)$ and the amount of MCC PH-101 $\left(\mathrm{X}_{2}\right)$ were chosen as independent variables in $3^{2}$ full factorial design, while $\%$ drug release at $10 \mathrm{~min}\left(\mathrm{Q}_{10 \%}\right)$ and time required for $80 \%$ drug release $\left(\mathrm{t}_{80}\right)$. were taken as dependent variables. Multiple linear regression analysis, ANOVA and graphical representation of the influence of factor by contour plots were performed using Sigmaplot 11.0. The experimental runs and measured responses of $3^{2}$ full factorial design batches of cefuroxime axetil dispersible tablets were depleted in table 6. [22-24].

\section{Stability study}

Optimized batch was packed in aluminum foil and was placed for stability study at $40^{\circ} \mathrm{C} / 75 \% \mathrm{RH}$ for $3 \mathrm{mo}$. Sample was evaluated after 3 mo for physical parameters and In vitro dissolution. The dissolution profile of the product was compared using the similarity factor, $\mathrm{f}_{2}$, which was calculated by the following formula.

$$
f_{2}=50 \log \left[\left\{1+\frac{1}{n} \sum_{t=1}^{n}\left(R_{t}-T_{t}\right)^{2}\right\}^{-0.5} x 100\right]
$$

where the log is logarithm to the base $10, \mathrm{n}$ is the number of time points, $\sum$ is summation over all time points, $R_{t}$ is the mean dissolution value of the reference profile at time $t$ and $T_{t}$ is the mean dissolution value of the test profile at the same time point. The USFDA draft guidance document contains more information on the similarity factor $\left(f_{2}\right)$. The value of the similarity factor $\left(f_{2}\right)$ between 50 and 100 suggests that the two dissolution profiles are similar [25-27].

Table 2: Formulation of factorial batches of cefuroxime axetil $125 \mathrm{mg}$ dispersible tablets

\begin{tabular}{|c|c|c|c|c|c|c|c|c|c|c|}
\hline S. No. & Ingredients (mg) & $F_{1}$ & $\mathbf{F}_{2}$ & $\mathbf{F}_{3}$ & $\mathbf{F}_{4}$ & $\mathbf{F}_{5}$ & $F_{6}$ & $\mathbf{F}_{7}$ & $\mathbf{F}_{8}$ & $F_{9}$ \\
\hline 1 & $\overline{\text { DRC }}$ & 543 & 543 & 543 & 543 & 543 & 543 & 543 & 543 & 543 \\
\hline 2 & SSG & 30 & 30 & 30 & 30 & 30 & 30 & 30 & 30 & 30 \\
\hline 3 & Mannitol & 15 & 15 & 15 & 25 & 25 & 25 & 35 & 35 & 35 \\
\hline 4 & MCC (PH 101) & 15 & 25 & 35 & 15 & 25 & 35 & 15 & 25 & 35 \\
\hline 5 & Sodium Saccharin & 20 & 20 & 20 & 20 & 20 & 20 & 20 & 20 & 20 \\
\hline 6 & Talc & 8 & 8 & 8 & 8 & 8 & 8 & 8 & 8 & 8 \\
\hline 7 & Sodium Stearyl Fumarate & 7 & 7 & 7 & 7 & 7 & 7 & 7 & 7 & 7 \\
\hline \multirow[t]{2}{*}{8} & Flavour & 5 & 5 & 5 & 5 & 5 & 5 & 5 & 5 & 5 \\
\hline & TOTAL & 643 & 653 & 663 & 653 & 663 & 673 & 663 & 673 & 683 \\
\hline
\end{tabular}

*Drug resin complex (DRC) contain $125 \mathrm{mg}$ cefuroxime axetil and kyron T-114 


\section{RESULTS AND DISCUSSION}

\section{Drug-excipient compatibility study}

Compatibility studies of pure drug with ion-exchange resin and other excipients were carried out prior to the preparation of taste masked dispersible tablet. Fig. 1 and fig. 2 shows DSC thermograms of cefuroxime axetil and kyron T-114, respectively. Fig. 3 and fig. 4 shows DSC thermograms of drug-resin complex and Drug-excipients

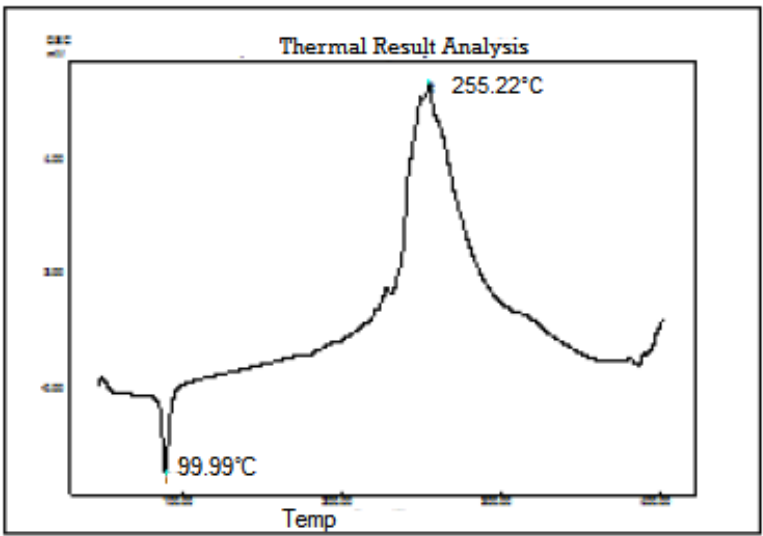

Fig. 1: DSC spectrum of cefuroxime axetil

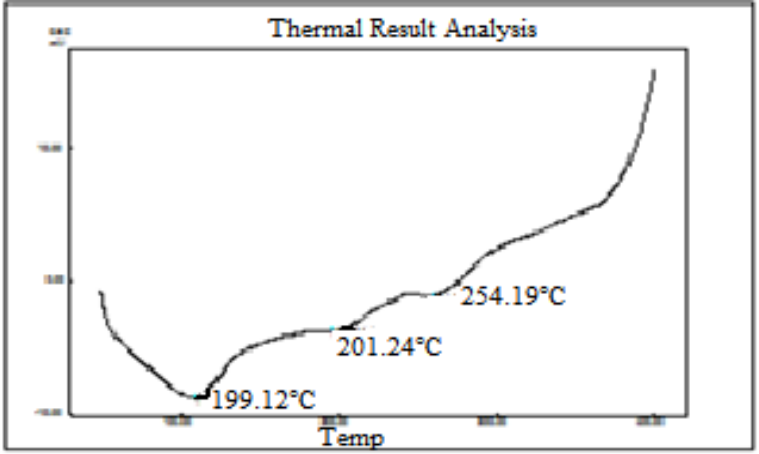

Fig. 3: DSC spectrum of drug-resin complex

\section{Evaluation of drug-resin complex (DRC)}

Trial batches of cefuroxime axetil-resin complex were prepared using different drug-resin ratio, different resins, $\mathrm{pH}$ and temperature. Taste and \% of drug loading of all the formulation were shown in table 3 . Indion-214 showed a higher percentage of physical mixture, respectively. The DSC analysis of the drug alone elicited a peak at $255.22^{\circ} \mathrm{C}$ and complex of cefuroxime axetil with kyron T-114 shows peak of drug at $256.78{ }^{\circ} \mathrm{C}$. Elicited peak of the physical mixture of DRC and other excipients was found to be at $254.16{ }^{\circ} \mathrm{C}$. Thus, it was thought to indicate that there was no evidence of interactions between cefuroxime axetil, kyron T-114 and the used excipients. So, it can conclude that drug and other excipients are compatible which each other [28, 29].

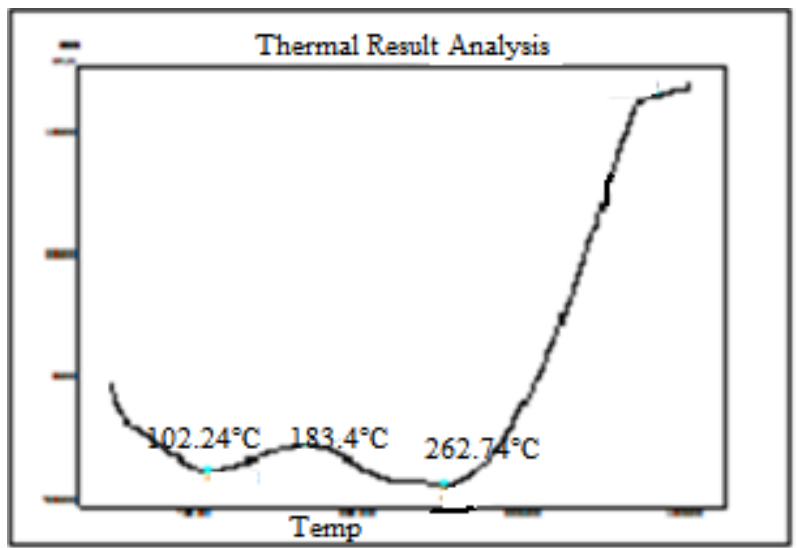

Fig. 2: DSC spectrum of kyron T-114

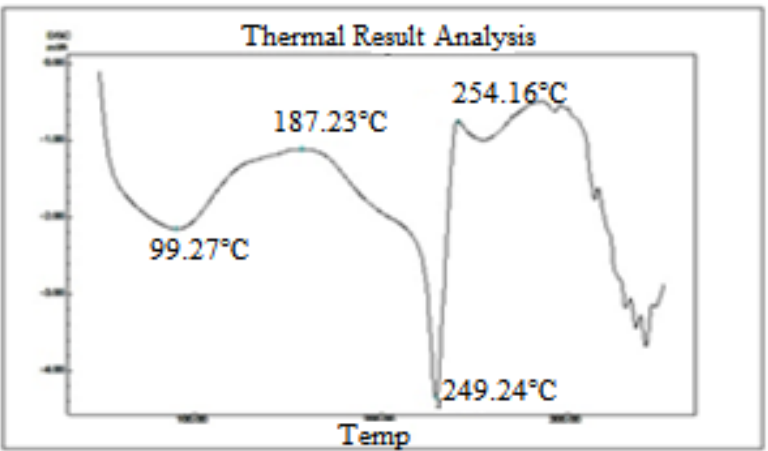

Fig. 4: DSC spectrum of physical mixture of drug-resin and excipients

drug loading but it cannot mask the bitter taste. The same condition was found with kyron T-134. Among the all batches DRC-9 showed less bitter taste and $92.70 \%$ of drug loading may be due to drug got ionised and cross-linked with kyron T-114 at pH 9. So, DRC-9 was use for further studies to prepare cefuroxime axetil dispersible tablet [30].

Table 3: Development and evaluation of drug-resin complex (DRC)

\begin{tabular}{|c|c|c|c|c|c|c|c|}
\hline Formulation & Resin & Ratio & pH & Time(hr) & Temp & Taste & \% Drug loading \\
\hline DRC-1 & Indion-214 & $1: 1$ & 7 & 2 & $30 \pm 0.5^{\circ} \mathrm{C}$ & ++++ & $59.21 \pm 1.3 \%$ \\
\hline DRC-2 & Indion 214 & $1: 2$ & 7 & 2 & $30 \pm 0.5^{\circ} \mathrm{C}$ & ++++ & $71.92 \pm 1.6 \%$ \\
\hline DRC-3 & Indion 214 & $1: 3$ & 9 & 3 & $30 \pm 0.5^{\circ} \mathrm{C}$ & ++++ & $82.24 \pm 0.8 \%$ \\
\hline DRC-4 & Indion 214 & $1: 3$ & 9 & 3 & $35 \pm 0.5^{\circ} \mathrm{C}$ & +++ & $87.73 \pm 0.4 \%$ \\
\hline DRC-5 & Kyron T-114 & $1: 1$ & 7 & 2 & $30 \pm 0.5^{\circ} \mathrm{C}$ & ++++ & $68.23 \pm 0.7 \%$ \\
\hline DRC-6 & Kyron T-114 & $1: 2$ & 7 & 2 & $30 \pm 0.5^{\circ} \mathrm{C}$ & ++++ & $77.49 \pm 1.4 \%$ \\
\hline DRC-7 & Kyron T-114 & $1: 3$ & 7 & 3 & $30 \pm 0.5^{\circ} \mathrm{C}$ & +++ & $84.27 \pm 1.3 \%$ \\
\hline DRC-8 & Kyron T-114 & $1: 3$ & 9 & 3 & $30 \pm 0.5^{\circ} \mathrm{C}$ & ++ & $88.39 \pm 0.7 \%$ \\
\hline DRC-9 & Kyron T-114 & $1: 3$ & 9 & 3 & $35 \pm 0.5^{\circ} \mathrm{C}$ & + & $92.70 \pm 0.3 \%$ \\
\hline DRC-11 & Dosion P551 & $1: 2$ & 7 & 3 & $30 \pm 0.5^{\circ} \mathrm{C}$ & ++++ & $62.80 \pm 1.5 \%$ \\
\hline DRC-10 & Kyron T 134 & $1: 1$ & 7 & 3 & $30 \pm 0.5^{\circ} \mathrm{C}$ & ++++ & $62.23 \pm 1.1 \%$ \\
\hline DRC-12 & Kyron T 134 & $1: 2$ & 7 & 3 & $30 \pm 0.5^{\circ} \mathrm{C}$ & +++ & $76.21 \pm 1.3 \%$ \\
\hline DRC-13 & Kyron T 134 & $1: 3$ & 7 & 3 & $35 \pm 0.5^{\circ} \mathrm{C}$ & +++ & $84.61 \pm 0.6 \%$ \\
\hline DRC-14 & Kyron T 134 & $1: 3$ & 9 & 3 & $3 \pm 0.55^{\circ} \mathrm{C}$ & +++ & $89.62 \pm 1.2 \%$ \\
\hline
\end{tabular}

$(n=6)$ 


\section{Preliminary trail batch evaluation of cefuroxime axetil $125 \mathbf{~ m g}$} dispersible tablets

Table 4 shows the results of pre-compression, post-compression and In vitro drug release at $45 \mathrm{~min}$ of preliminary trail batches of dispersible tablets. The hardness and friability of all the formulation was found to be in a range of 1.9 to $3.9 \mathrm{~kg} / \mathrm{cm}^{3}$ and 0.30 to 0.70 respectively. Trail batches of dispersible tablets indicate that hardness dependent on MCC and mannitol concentration. Batch X5 and $\mathrm{X} 6$ shows less disintegration time compared to batch X1, X2, X3 and X4. Croscarmellose Sodium was increased in batch X7 but it will not impact on disintegration time. So, sodium starch glycolate was used as super disintegrants for further Preliminary trial batch. Sodium starch glycolate further increase in batch X11 and X12 but it decreases hardness of tablet and less impact on disintegration time. In Batch X13 and X14; MCC and mannitol was taken as filler for tablet. X13, X14 gave $94.61 \%$ and $89.65 \%$ drug release in $45 \mathrm{~min}$, respectively. The results of preliminary study revealed that MCC or mannitol alone was not sufficient to achieve the desired release profile. Hence, further trials were carried out using combination MCC and mannitol in order to understand their effect and to optimize concentration of both for desired release profile [31,32].

Table 4: Preliminary trail batch evaluation of cefuroxime axetil $125 \mathrm{mg}$ dispersible tablets

\begin{tabular}{|c|c|c|c|c|c|c|c|c|c|c|c|c|c|c|c|}
\hline $\begin{array}{l}\text { S. } \\
\text { N } \\
\text { o. }\end{array}$ & $\begin{array}{l}\text { Ingred } \\
\text { ients }\end{array}$ & $\mathrm{X} 1$ & $\mathrm{X} 2$ & X3 & $X 4$ & $\mathrm{X5}$ & X6 & X7 & X8 & X9 & $\mathrm{X10}$ & $\mathrm{X} 11$ & $\mathrm{X} 12$ & X13 & $\mathrm{X14}$ \\
\hline \multirow[t]{2}{*}{1} & Angle & 30.96 & 29.98 & 29.05 & 27.75 & 26.56 & 27.34 & 25.82 & 28.61 & 27.34 & 25.82 & 27.75 & 28.17 & 29.05 & 29.51 \\
\hline & $\begin{array}{l}\text { of } \\
\text { repose } \\
\left({ }^{\circ}\right)\end{array}$ & \pm 1.47 & \pm 1.22 & \pm 1.87 & \pm 0.56 & $\begin{array}{l} \pm \\
1.36\end{array}$ & \pm 1.58 & \pm 0.86 & \pm 1.47 & \pm 1.65 & \pm 1.23 & \pm 1.32 & \pm 1.38 & \pm 1.24 & \pm 1.60 \\
\hline \multirow[t]{2}{*}{2} & Bulk & 0.41 & 0.42 & 0.40 & 0.43 & 0.39 & 0.42 & 0.38 & 0.45 & 0.42 & 0.40 & 0.39 & 0.40 & 0.41 & 0.39 \\
\hline & density & \pm 0.07 & \pm 0.03 & \pm 0.03 & \pm 0.02 & \pm 0.04 & \pm 0.06 & \pm 0.07 & \pm 0.05 & \pm 0.04 & \pm 0.05 & \pm 0.06 & \pm 0.09 & \pm 0.08 & \pm 0.03 \\
\hline \multirow[t]{2}{*}{3} & Tapped & 0.58 & 0.58 & 0.57 & 0.59 & 0.56 & 0.57 & 0.57 & 0.61 & 0.58 & 0.57 & 0.57 & 0.58 & 0.57 & 0.57 \\
\hline & density & \pm 0.01 & \pm 0.02 & \pm 0.03 & \pm 0.02 & \pm 0.03 & \pm 0.03 & \pm 0.03 & \pm 0.01 & \pm 0.01 & \pm 0.03 & \pm 0.04 & \pm 0.03 & \pm 0.01 & \pm 0.03 \\
\hline \multirow[t]{2}{*}{4} & Carr's & 30.10 & 28.06 & 28.72 & 26.80 & 29.13 & 25.92 & 32.99 & 25.85 & 27.54 & 29.36 & 32.30 & 31.12 & 27.98 & 30.65 \\
\hline & index & \pm 1.17 & \pm 1.35 & \pm 2.12 & \pm 1.34 & \pm 0.57 & \pm 2.21 & \pm 2.11 & \pm 1.22 & \pm 2.17 & \pm 2.01 & \pm 1.37 & \pm 0.45 & \pm 0.78 & \pm 0.87 \\
\hline \multirow[t]{2}{*}{5} & Hausne & 1.40 & 1.39 & 1.40 & 1.37 & 1.41 & 1.35 & 1.49 & 1.35 & 1.38 & 1.42 & 1.48 & 1.45 & 1.39 & 1.44 \\
\hline & $\begin{array}{l}\text { r's ratio } \\
\text { ratio }\end{array}$ & \pm 0.12 & \pm 0.10 & \pm 0.13 & \pm 0.03 & \pm 0.04 & \pm 0.11 & \pm 0.13 & \pm 0.03 & \pm 0.04 & \pm 0.05 & \pm 0.14 & \pm 0.07 & \pm 0.08 & \pm 0.04 \\
\hline \multirow[t]{3}{*}{6} & Avg. & 654.4 & 653.2 & 657.3 & 653.6 & 653.2 & 651.5 & 650.9 & 656.2 & 650.1 & 654.4 & 651.3 & 670.4 & 661.6 & 651.4 \\
\hline & weight & 4 & 4 & 2 & 7 & 4 & 7 & 2 & 7 & 1 & 7 & 5 & & \pm 8.45 & \\
\hline & (mg) & \pm 6.67 & \pm 5.25 & \pm 5.49 & \pm 4.46 & \pm 6.46 & \pm 3.56 & \pm 4.18 & \pm 4.57 & \pm 4.87 & \pm 1.48 & \pm 5.47 & \pm 7.45 & & \pm 6.45 \\
\hline \multirow[t]{2}{*}{7} & \% Drug & 98.17 & 98.58 & 97.41 & 99.27 & 99.25 & $98 \pm$ & 98.65 & 96.44 & 95.4 & 99.17 & 99.58 & 98.41 & 98.27 & 99.25 \\
\hline & content & \pm 0.33 & \pm 1.32 & \pm 0.83 & \pm 1.02 & \pm 0.41 & 0.90 & \pm 0.70 & \pm 0.56 & \pm 0.22 & \pm 0.33 & \pm 1.32 & \pm 0.83 & \pm 1.02 & \pm 0.41 \\
\hline \multirow[t]{2}{*}{8} & Hardne & $3.61 \pm$ & $2.93 \pm$ & $3.23 \pm$ & $3.03 \pm$ & $3.32 \pm$ & $2.58 \pm$ & $3.68 \pm$ & $3.65 \pm$ & $2.85 \pm$ & $3.67 \pm$ & $2.97 \pm$ & $1.95 \pm$ & $3.25 \pm$ & $3.25 \pm$ \\
\hline & $\begin{array}{l}\text { ss } \\
(\mathrm{kg} / \mathrm{cm} \\
2)\end{array}$ & 0.72 & 0.44 & 0.32 & 0.41 & 0.32 & 0.75 & 0.55 & 0.22 & 0.43 & 0.25 & 0.35 & 0.48 & 0.42 & 0.6 . \\
\hline \multirow[t]{2}{*}{9} & Thickn & $7.35 \pm$ & $7.42 \pm$ & $7.33 \pm$ & 7.4 & $7.45 \pm$ & $7.39 \pm$ & 7.3 & $7.29 \pm$ & $7.44 \pm$ & $7.36 \pm$ & $7.57 \pm$ & $7.63 \pm$ & $7.59 \pm$ & $7.41 \pm$ \\
\hline & $\begin{array}{l}\text { ess } \\
(\mathrm{mm})\end{array}$ & 0.23 & 0.23 & 0.15 & \pm 0.31 & 0.15 & 0.32 & \pm 0.35 & 0.49 & 0.35 & 0.23 & 0.15 & 0.22 & 0.31 & 0.11 \\
\hline 1 & Friabili & 0.39 & 0.63 & 0.36 & 0.45 & 0.74 & 0.73 & 0.55 & 0.35 & 0.59 & 0.74 & 0.69 & 0.35 & 0.52 & 0.71 \\
\hline 0 & ty $(\%)$ & \pm 0.07 & \pm 0.06 & \pm 0.01 & \pm 0.04 & \pm 0.02 & \pm 0.04 & \pm 0.05 & \pm 0.05 & \pm 0.01 & \pm 0.06 & \pm 0.08 & \pm 0.06 & \pm 0.02 & \pm 0.03 \\
\hline 1 & Wettin & $14 \pm 1$ & $13 \pm 2$ & $13 \pm 1$ & $11 \pm 1$ & $12 \pm 2$ & $12 \pm 2$ & $11 \pm 1$ & $12 \pm 2$ & $11 \pm 1$ & $10 \pm 1$ & $9 \pm 2$ & $8 \pm 1$ & $9 \pm 2$ & $11 \pm 1$ \\
\hline 1 & g time & & & & & & & & & & & & & & \\
\hline 1 & Disinte & 30 & 27 & 29 & 26 & 20 & 18 & 17 & 14 & 15 & 15 & 13 & 12 & 14 & 19 \\
\hline 2 & $\begin{array}{l}\text { gration } \\
\text { time }\end{array}$ & \pm 1 & \pm 2 & \pm 1 & \pm 2 & \pm 1 & \pm 2 & \pm 2 & \pm 1 & \pm 2 & \pm 2 & \pm 1 & \pm 1 & \pm 1 & \pm 2 \\
\hline $\begin{array}{l}1 \\
3\end{array}$ & $\begin{array}{l}\text { Finenes } \\
\text { s of } \\
\text { dispers } \\
\text { ion }\end{array}$ & Fail & Fail & Fail & Pass & Pass & Pass & Pass & Pass & Pass & Pass & Pass & Pass & Pass & Pass \\
\hline 1 & In vitro & 42.09 & 75.18 & 54.09 & 76.27 & 63.52 & 78.03 & 83.54 & 88.49 & 88.73 & 92.08 & 89.62 & 92.12 & 94.61 & 89.65 \\
\hline \multirow[t]{2}{*}{4} & drug & $9 \pm 2.2$ & $7 \pm 2.0$ & $7 \pm 2.3$ & $5 \pm 2.3$ & $0 \pm 2.1$ & $8 \pm 1.9$ & $4 \pm 1.0$ & $4 \pm 2.3$ & $9 \pm 2.3$ & $6 \pm 2.3$ & $8 \pm 1.9$ & $1 \pm 2.3$ & $9 \pm 2.1$ & $0 \pm 2.3$ \\
\hline & $\begin{array}{l}\text { release } \\
\text { at } 45 \\
\min \end{array}$ & 2 & 9 & 2 & 4 & 1 & 7 & 5 & 2 & 5 & 2 & 0 & 6 & 3 & 6 \\
\hline
\end{tabular}

$(n=3)$

\section{$3^{2}$ full factorial design model evaluation}

A statistical model incorporating interactive and polynomial terms was used to evaluate the responses:

\section{$\mathrm{Y}=\mathrm{b}_{0+} \mathrm{b}_{1} \mathrm{X}_{1}+\mathrm{b}_{2} \mathrm{X}_{2}+\mathrm{b}_{11} \mathrm{X}_{1}{ }^{2}+\mathrm{b}_{22} \mathrm{X}_{2}^{2}+\mathrm{b}_{12} \mathrm{X}_{1} \mathrm{X}_{2}$}

where, $\mathrm{Y}$ is the dependent variable, $\mathrm{b}_{\mathrm{o}}$ is the arithmetic mean response of the 9 runs and any bi is the estimated coefficients for the related factor $\mathrm{Xi}$. The main effects $\left(\mathrm{X}_{1}\right.$ and $\left.\mathrm{X}_{2}\right)$ represent the average result of changing one factor at a time from its low to high value. The polynomial terms $\left(\mathrm{X}_{1}{ }^{2}\right.$ and $\left.\mathrm{X}_{2}{ }^{2}\right)$ are included to investigate nonlinearity. The interaction term " $\mathrm{X}_{1} \mathrm{X}_{2}$ " shows how the response changes when the two factors change simultaneously. Evaluation data of pre-compression and post-compression parameters of factorial batches and In vitro \% drug release were presented in table
5 and table 6 . Table 7 describes the effect of independent variables on dependent variables by $3^{2}$ full factorial designs. The fitted equations (full model) relating the responses that is \% drug release at $10 \mathrm{~min}\left(\mathrm{Q}_{10 \%}\right)$ and time required for $80 \%$ drug release $(\mathrm{min})\left(\mathrm{T}_{80}\right)$ to the transformed factor were shown in table 8. The polynomial equation can be used to draw conclusion after considering the magnitude of coefficient and the mathematical sign it carries (i.e. positive or negative). The results of ANOVA suggested that $F$ values calculated for $\mathrm{Q}_{10} \%$ and $\mathrm{t}_{80} \%$ are 59.668 and 92.625 , respectively (table 8). Tabulated $F$ value was found to be 9.013 at $\alpha=0.05$. Calculated $F$ values are greater than tabulated for all dependent variables therefore factors selected have shown significant effects. From the results of multiple regression analysis, it was found that both factors had statistically significant influence on all dependent variables as $\mathrm{p}<0.05[33,34]$. 
Table 5: Factorial batch evaluation of cefuroxime axetil $125 \mathrm{mg}$ dispersible tablets

\begin{tabular}{|c|c|c|c|c|c|c|c|c|c|c|}
\hline S. No. & Ingredients & F1 & F2 & F3 & F4 & F5 & F6 & F7 & F8 & F9 \\
\hline \multirow[t]{2}{*}{1} & Angle of repose $\left({ }^{\circ}\right)$ & 25.11 & 25.82 & 27.34 & 23.8 & 25.11 & 27.75 & 27.75 & 28.61 & 29.05 \\
\hline & & \pm 0.68 & \pm 1.21 & \pm 1.21 & \pm 0.38 & \pm 0.58 & \pm 0.44 & \pm 0.57 & \pm 0.46 & \pm 1.11 \\
\hline \multirow[t]{2}{*}{2} & Bulk density & 0.40 & 0.38 & 0.39 & 0.41 & 0.42 & 0.42 & 0.40 & 0.42 & 0.41 \\
\hline & & \pm 0.02 & \pm 0.01 & \pm 0.03 & \pm 0.05 & \pm 0.03 & \pm 0.06 & \pm 0.07 & \pm 0.03 & \pm 0.01 \\
\hline \multirow[t]{2}{*}{3} & Tapped density & 0.57 & 0.56 & 0.58 & 0.57 & 0.59 & 0.60 & 0.57 & 0.61 & 0.59 \\
\hline & & \pm 0.03 & \pm 0.01 & \pm 0.02 & \pm 0.02 & \pm 0.01 & \pm 0.04 & \pm 0.02 & \pm 0.03 & \pm 0.04 \\
\hline \multirow[t]{2}{*}{4} & Carr's index & 29.60 & 31.99 & 32.14 & 27.85 & 29.01 & 29.21 & 29.71 & 30.73 & 30.82 \\
\hline & & \pm 1.18 & \pm 0.29 & \pm 1.12 & \pm 1.19 & \pm 1.15 & \pm 0.57 & \pm 0.67 & \pm 0.87 & \pm 1.18 \\
\hline \multirow[t]{2}{*}{5} & Hausner's ratio & 1.42 & 1.47 & 1.47 & 1.39 & 1.41 & 1.41 & 1.42 & 1.44 & 1.45 \\
\hline & & \pm 0.11 & \pm 0.15 & \pm 0.09 & \pm 0.08 & \pm 0.13 & \pm 0.23 & \pm 0.12 & \pm 0.14 & \pm 0.11 \\
\hline \multirow[t]{2}{*}{6} & Avg. weight (mg) & 641.45 & 654.65 & 61.56 & 652.08 & 664.42 & 671.83 & 662.65 & 671.19 & 681.34 \\
\hline & & \pm 3.44 & \pm 3.65 & \pm 4.35 & \pm 5.23 & \pm 4.66 & \pm 5.57 & \pm 4.64 & \pm 5.17 & \pm 2.64 \\
\hline \multirow[t]{2}{*}{7} & $\%$ Drug content & 98.27 & 99.2 & 97.13 & 99.27 & 96.65 & 97.49 & 98.45 & 99.35 & 98.24 \\
\hline & & \pm 0.31 & \pm 0.13 & \pm 0.22 & \pm 0.24 & \pm 0.55 & \pm 1.43 & \pm 0.53 & \pm 0.67 & \pm 0.56 \\
\hline \multirow[t]{2}{*}{8} & Hardness $\left(\mathrm{kg} / \mathrm{cm}^{2}\right)$ & 3.62 & 2.93 & 3.35 & 2.84 & 3.22 & 3.14 & 2.74 & 3.35 & 3.25 \\
\hline & & \pm 0.32 & \pm 0.31 & \pm 0.67 & \pm 0.56 & \pm 0.53 & \pm 0.62 & \pm 0.51 & \pm 0.45 & \pm 0.25 \\
\hline \multirow[t]{2}{*}{9} & Thickness (mm) & 6.61 & 6.57 & 6.65 & 6.74 & 6.63 & 6.87 & 6.76 & 6.73 & 6.87 \\
\hline & & \pm 0.13 & \pm 0.25 & \pm 0.23 & \pm 0.42 & \pm 0.54 & \pm 0.34 & \pm 0.53 & \pm 0.13 & \pm 0.45 \\
\hline \multirow[t]{2}{*}{10} & Friability (\%) & 0.67 & 0.78 & 0.47 & 0.88 & 0.72 & 0.49 & 0.54 & 0.64 & 0.68 \\
\hline & & \pm 0.04 & \pm 0.08 & \pm 0.02 & \pm 0.07 & \pm 0.03 & \pm 0.06 & \pm 0.04 & \pm 0.05 & \pm 0.08 \\
\hline 11 & Wetting time & $18 \pm 1$ & $17 \pm 2$ & $14 \pm 1$ & $13 \pm 1$ & $11 \pm 2$ & $10 \pm 2$ & $8 \pm 1$ & $10 \pm 1$ & $12 \pm 2$ \\
\hline 12 & Disintegration time & $14 \pm 1$ & $15 \pm 2$ & $17 \pm 1$ & $13 \pm 1$ & $15 \pm 2$ & $16 \pm 2$ & $12 \pm 1$ & $13 \pm 2$ & $14 \pm 1$ \\
\hline 13 & Fineness of dispersion & Pass & Pass & Pass & Pass & Pass & Pass & Pass & Pass & Pass \\
\hline
\end{tabular}

$(\mathrm{n}=6)$

Table 6: In vitro dissolution of factorial batches

\begin{tabular}{|c|c|c|c|c|c|c|c|c|c|}
\hline Time (min) & F1 & F2 & F3 & F4 & F5 & F6 & F7 & F8 & F9 \\
\hline 0 & 0 & 0 & 0 & 0 & 0 & 0 & 0 & 0 & 0 \\
\hline \multirow[t]{2}{*}{5} & 49.78 & 51.02 & 50.61 & 54.62 & 57.19 & 55.65 & 59.66 & 56.26 & 54.93 \\
\hline & \pm 2.31 & \pm 1.96 & \pm 2.42 & \pm 1.73 & \pm 1.85 & \pm 2.08 & \pm 2.19 & \pm 2.19 & \pm 1.73 \\
\hline \multirow[t]{2}{*}{10} & 58.78 & 59.36 & 60.18 & 64.61 & 66.47 & 68.83 & 69.15 & 68.63 & 66.16 \\
\hline & \pm 2.09 & \pm 2.32 & \pm 2.09 & \pm 1.97 & \pm 1.05 & \pm 2.09 & \pm 2.32 & \pm 2.32 & \pm 1.97 \\
\hline \multirow[t]{2}{*}{15} & 62.93 & 65.15 & 65.15 & 71.44 & 72.89 & 72.79 & 74.55 & 71.25 & 70.73 \\
\hline & \pm 2.22 & \pm 2.68 & \pm 2.33 & \pm 2.21 & \pm 2.09 & \pm 2.45 & \pm 2.10 & \pm 2.22 & \pm 2.21 \\
\hline \multirow[t]{2}{*}{20} & 69.90 & 72.08 & 72.08 & 77.67 & 79.33 & 78.81 & 81.19 & 75.42 & 75.41 \\
\hline & \pm 2.36 & \pm 2.12 & \pm 2.24 & \pm 2.34 & \pm 2.34 & \pm 2.11 & \pm 2.12 & \pm 2.46 & \pm 2.34 \\
\hline \multirow[t]{2}{*}{25} & 77.86 & 75.84 & 77.69 & 82.47 & 85.88 & 85.37 & 89.19 & 80.42 & 80.51 \\
\hline & \pm 2.13 & \pm 2.36 & \pm 2.13 & \pm 2.24 & \pm 1.89 & \pm 1.49 & \pm 2.47 & \pm 1.90 & \pm 2.24 \\
\hline \multirow[t]{2}{*}{30} & 81.49 & 82.49 & 84.24 & 86.15 & 89.46 & 87.71 & 93.09 & 87.59 & 87.17 \\
\hline & \pm 2.25 & \pm 1.91 & \pm 2.16 & \pm 2.13 & \pm 2.13 & \pm 2.26 & \pm 2.25 & \pm 2.02 & \pm 2.13 \\
\hline \multirow[t]{2}{*}{45} & 83.54 & 87.50 & 88.75 & 90.86 & 92.95 & 89.66 & 96.38 & 93.03 & 91.68 \\
\hline & \pm 2.45 & \pm 2.10 & \pm 2.09 & \pm 2.32 & \pm 2.46 & \pm 2.36 & \pm 2.12 & \pm 2.32 & \pm 1.97 \\
\hline
\end{tabular}

$(n=6)$

Table 7: Runs and measured responses of $3^{2}$ factorial design batches

\begin{tabular}{|c|c|c|c|c|}
\hline $\begin{array}{l}\text { Batch } \\
\text { code }\end{array}$ & $\begin{array}{l}\text { Spray rate } \\
\left(\mathrm{X}_{1}\right)\end{array}$ & $\begin{array}{l}\text { Inlet air temperature } \\
\left(\mathrm{X}_{2}\right)\end{array}$ & $\begin{array}{l}\text { \% Drug release at } 10 \mathrm{~min}\left(\mathrm{Q}_{10 \%}\right) \\
\mathrm{Y}_{1}\end{array}$ & $\begin{array}{l}\text { Time required for } 80 \% \text { drug release }(\mathrm{min})\left(\mathrm{T}_{80}\right) \\
\mathrm{Y}_{2}\end{array}$ \\
\hline F1 & -1 & -1 & $60.18 \pm 1.09$ & $31 \pm 2$ \\
\hline $\mathrm{F} 2$ & 0 & -1 & $59.53 \pm 0.49$ & $32 \pm 1$ \\
\hline F3 & 1 & -1 & $58.36 \pm 0.85$ & $33 \pm 1$ \\
\hline F4 & -1 & 0 & $68.83 \pm 0.77$ & $26 \pm 2$ \\
\hline F5 & 0 & 0 & $67.47 \pm 0.69$ & $26 \pm 1$ \\
\hline F6 & 1 & 0 & $64.61 \pm 1.07$ & $27 \pm 2$ \\
\hline F7 & -1 & 1 & $69.14 \pm 1.09$ & $25 \pm 1$ \\
\hline F8 & 0 & 1 & $67.44 \pm 0.59$ & $26 \pm 1$ \\
\hline F9 & 1 & 1 & $66.15 \pm 0.55$ & $27 \pm 2$ \\
\hline \multicolumn{5}{|c|}{ Factors and the levels in the design } \\
\hline \multicolumn{2}{|c|}{ Independent variables } & $\operatorname{Low}(-1)$ & Medium (0) & High (1) \\
\hline \multicolumn{2}{|c|}{ Mannitol $\left(\mathrm{X}_{1}\right)$} & 15 & 25 & 35 \\
\hline \multicolumn{2}{|c|}{ MCC PH-101 $\left(\mathrm{X}_{2}\right)$} & 15 & 25 & 35 \\
\hline
\end{tabular}

$(n=6)$

Full and reduced model for $\%$ drug release at $10 \mathrm{~min}$

$\mathrm{Q}_{10 \%}=67.153-\left(1.505 \mathrm{X}_{1}\right)+\left(4.111 \mathrm{X}_{2}\right)-\left(0.294 \mathrm{X}_{1}^{2}\right)-\left(0.270 \mathrm{X}_{2}^{2}\right)-(3.501$ $\left.\mathrm{X}_{1} \mathrm{X}_{2}\right)$
From the 3D plot (fig. 5) and the regression coefficient values of factors, it was concluded that when MCC PH-101 concentration was increase that time drug release also increase due to its hydrophilic 
nature. For drug release at $10 \mathrm{~min}$, the significance levels of the coefficients $b_{11}$ and $b_{22}$ were found to be $P=0.454$, and 0.617 repectively, so they were omitted from the full model to generate a reduced model. The coefficients $b_{0}, b_{1}, b_{2}$ and $b_{12}$ significant at $\mathrm{P}<0.05$; hence they were retained in the reduced model. $[35,36]$ The reduced model for drug release at $10 \mathrm{~min}$ :

$$
Q_{10 \%}=67.153-\left(1.505 X_{1}\right)+\left(4.111 X_{2}\right)-\left(3.501 X_{1} X_{2}\right)
$$

Full and reduced model for time required for $\mathbf{8 0 \%}$ drug release

$$
\mathrm{T}_{80}=26.611+\left(0.833 \mathrm{X}_{1}\right)-\left(2.917 \mathrm{X}_{2}\right)-\left(-0.000 \mathrm{X}_{1}^{2}\right)-\left(0.167 \mathrm{X}_{2}^{2}\right)+(2.583
$$$$
\left.\mathrm{X}_{1} \mathrm{X}_{2}\right)
$$

From the 3D plot graph (fig. 6) and the regression coefficient values of factors, it was concluded that when mannitol concentration was increase that time drug release decrease may be due to its very low hydrophilic nature. For time required for $80 \%$ drug release, the significance levels of the coefficients $\mathrm{b}_{11}$ and $\mathrm{b}_{22}$ were found to be $\mathrm{P}=$ 1.00 , and 0.584 repectively, so they were omitted from the full model to generate a reduced model. The coefficients $b_{0}, b_{1}, b_{2}$ and $b_{12}$ significant at $\mathrm{P}<0.05$; hence they were retained in the reduced model. $[37,38]$ The reduced model for Drug release time required for $80 \%$ drug release:

$\mathrm{T}_{80}=26.611+\left(0.833 \mathrm{X}_{1}\right)-\left(2.917 \mathrm{X}_{2}\right)+\left(2.583 \mathrm{X}_{1} \mathrm{X}_{2}\right)$

Table 8: Results of the ANOVA for dependent variables

\begin{tabular}{lllll}
\hline Source of variation & DF & SS & MS & F value \\
\hline Q $_{10}$ Dependent variable & & & 27.995 & P value \\
Regression & 5 & 139.975 & 0.469 & 59.668 \\
Residual & 3 & 1.408 & 17.673 & 0.003 \\
Total & 8 & 141.382 & & \\
t80 Dependent variable & & & 13.722 & 92.625 \\
Regression & 5 & 68.611 & 0.148 & 0.002 \\
Residual & 3 & 0.444 & 8.632 & \\
Total & 8 & 69.056 & \\
\hline
\end{tabular}

\begin{tabular}{|c|c|c|c|c|c|c|c|c|}
\hline \multirow[t]{2}{*}{ Responses } & \multirow[t]{2}{*}{ Model } & \multicolumn{7}{|c|}{ Coefficient of regression parameters } \\
\hline & & $\mathbf{b}_{0}$ & $\mathbf{b}_{1}$ & $\mathbf{b}_{2}$ & $\mathbf{b}_{11}$ & $\mathbf{b}_{22}$ & $\mathbf{b}_{12}$ & $\mathbf{R}^{2}$ \\
\hline \multirow{2}{*}{$\mathrm{Q}_{10 \%}$} & Full & 67.153 & -1.505 & 4.111 & 0.294 & -0.270 & -3.501 & 0.990 \\
\hline & Reduced & 67.153 & -1.505 & 4.111 & - & - & -3.501 & - \\
\hline \multirow[t]{2}{*}{$\mathrm{T}_{80}$} & Full & 26.611 & 0.833 & -2.917 & -0.000 & -0.167 & 2.583 & 0.994 \\
\hline & Reduced & 26.611 & 0.833 & -2.917 & - & - & 2.583 & - \\
\hline
\end{tabular}

Table 9: Summary of regression output of factors for measured responses

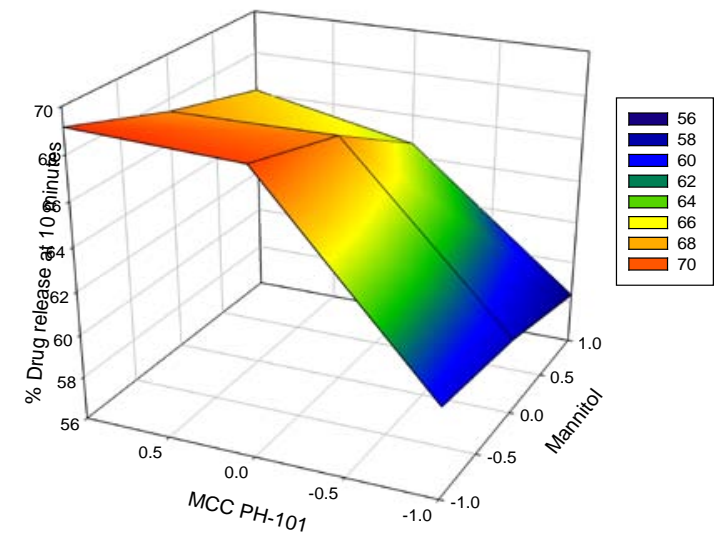

Fig. 5: 3D ssplot showing the effect of conc. of mannitol $\left(X_{1}\right)$ and MCC PH-101 $\left(\mathrm{X}_{2}\right)$ on \% drug release at $10 \mathrm{~min}$

\section{Formulation of check point batch}

To validate the evolved mathematical models, a check point batches CP1 was prepared and evaluated. The observed and predicted values for batch CP1 were shown in table 10. Good correlation was found between observed and predicted values shown in table 11 . Hence, it was concluded that the evolved models may be used for theoretical prediction of responses within the factor space [22].

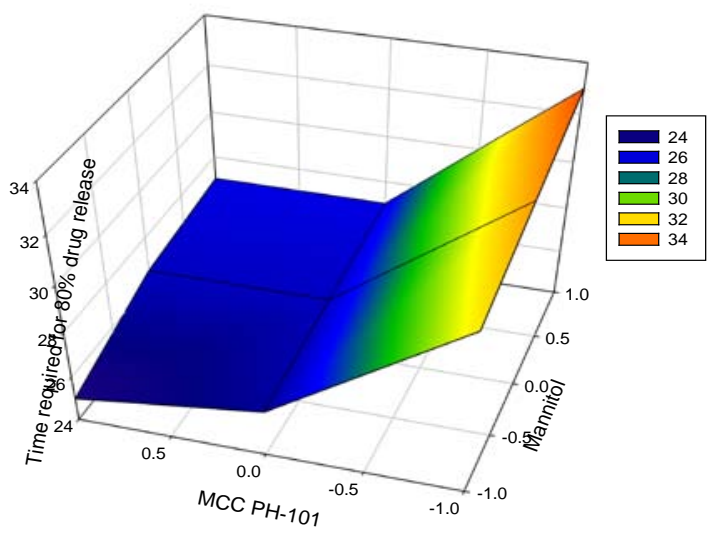

Fig. 6: 3D plot showing the effect of conc. of mannitol $\left(X_{1}\right)$ and MCC $\mathrm{PH}-101\left(\mathrm{X}_{2}\right)$ on time required for $80 \%$ drug release

\section{Selection of optimized batch in factorial design study}

In the present study, the following constraints were arbitrarily used for the selection of an optimized batch: $\mathrm{Q}_{10}>65 \%$ and $\mathrm{T}_{80 \%}<30 \mathrm{~min}$. Batches $\mathrm{F}_{4}, \mathrm{~F}_{5}, \mathrm{~F}_{7}, \mathrm{~F}_{8}$ and $\mathrm{F}_{9}$ met the selection criteria. Batch $\mathrm{F}_{7}$ showed higest $\%$ drug release at $10 \mathrm{~min}(69.149)$ and lowest time required for $80 \%$ drug release ( $25 \mathrm{~min}$ ), Hence, Batch $\mathrm{F}_{7}$ was selected as an optimized batch. The optimized formulation was subjected to accelerated stability study.

Table 10: Formulation and evaluation of check point batches

\begin{tabular}{lllll}
\hline Batch code & \multicolumn{3}{l}{ Variable level } & Actual value \\
\cline { 2 - 5 } & Coded value & & $\mathbf{X}_{\mathbf{1}}(\mathbf{m g})$ & $\mathbf{X}_{2}(\mathbf{m l})$ \\
\cline { 2 - 4 } & $\mathbf{X}_{\mathbf{1}}$ & -0.5 & 20 & 20 \\
\hline CP1 & -0.5 & & 20 \\
\hline
\end{tabular}


Table 11: Of check point batches and comparison with predicted value

\begin{tabular}{lll}
\hline Parameter & Actual value & Predicted value \\
\hline$\%$ drug release at $10 \mathrm{~min}\left(\mathrm{Q}_{10 \%}\right)$ & $63.54 \pm 1.35$ & 64.99 \\
Time required for $80 \%$ drug release $\left(\mathrm{T}_{80}\right)$ & $27 \pm 1$ & 28.63 \\
\hline
\end{tabular}

$(n=6)$

Table 12: Comparison of Evaluation parameters of optimized batch $F_{7}$ under stability study

\begin{tabular}{llll}
\hline S. No. & Parameters & Initial & After 3 mo \\
\hline 1 & Hardness $\left(\mathrm{kg} / \mathrm{cm}^{2}\right)$ & $2.74 \pm 0.51$ & $2.55 \pm 0.33$ \\
2 & Average weight $(\mathrm{mg})$ & $662.65 \pm 4.64$ & $660.15 \pm 2.11$ \\
3 & Disintegration time (min) & $12 \pm 1$ & $13 \pm 2$ \\
\hline
\end{tabular}

$(n=6)$

\section{Short term stability study}

Batch $\mathrm{F}_{7}$ was kept for stability study. The in vitro release profile at initial and after 3 mo was compared using similarity factor, $f_{2}$, value which was found to be 86.50 . There is no significant difference in similarity factor. Evaluation parameter of stability parameter was shown in table 12 .

\section{CONCLUSION}

The objective of the present investigation was to formulate, evaluate and optimize the cefuroxime axetil $125 \mathrm{mg}$ dispersible tablets to achieve quick disintegration and fast release of the drug for paediatric patients. Kyron T-114 was used as teste masking agent that showed highest \% of drug loading and test masking. These formulations were evaluated for the parameters like drug excipient compatibility study, thickness, hardness, weight variation, \% friability, disintegration test, in vitro drug release and accelerated stability studies. On the basis of preliminary results, the amount of mannitol $\left(\mathrm{X}_{1}\right)$ and the amount of MCC PH-101 $\left(\mathrm{X}_{2}\right)$ were chosen as independent variables in $3^{2}$ full factorial design, while \% drug release at $10 \mathrm{~min}\left(\mathrm{Q}_{10 \%}\right)$ and time required for $80 \%$ drug release $\left(\mathrm{t}_{80}\right)$, were taken as dependent variables. Multiple linear regression analysis, ANOVA and graphical representation of the influence of factor by contour plots were performed using Sigmaplot 11.0. From the results of multiple regression analysis, it was found that both factors had significant influence on all dependent variables. Check point batch was prepared to validate the evolved model. Batch $\mathrm{F}_{7}$ was selected as an optimized batch and it was found to be stable in the stability evaluation.

\section{AUTHORS CONTRIBUTIONS}

All the author have contributed equally

\section{CONFLICT OF INTERESTS}

Declared none

\section{REFERENCES}

1. Deshkar SS, Pawara AS, Shirolkar SV. Formulation and optimization of floating tablets of clopidogrel bisulphate using design of experiments. Int J Appl Pharm 2018;10:126-34.

2. Abdelbary G, Prinderre P, Eouani C, Joachim J, Reynier JP, Piccerelle $P$. The preparation of orally disintegrating tablets using a hydrophilic waxy binder. Int J Pharm 2004;278:423-33.

3. Patel BP, Patel JK, Rajput GC, Thakor RS. Formulation and evaluation of mouth dissolving tablets of cinnarizine. Indian J Pharm Sci 2010;72:522-5.

4. Al-dhahir RK, Al-kotaji M. Formulation of orally disintegrating tablets of cinnarizine by using direct compression method. Int J Appl Pharm 2018;11:117-23.

5. Bhowmik D, Chiranjib B, Chandira RM. Fast dissolving tablet: an overview. J Chem Pharm Res 2009;1:163-77.

6. Sharma S, Lewis S. Taste masking technologies: a review. Int J Pharm Pharm Sci 2010;2:6-13.

7. Nanda A, Kandarapu R, Garg S. An update on taste masking technologies for oral pharmaceuticals. Indian J Pharm Sci 2002;64:10-7.
8. Sohi H, Sultana Y, Khar R. Taste masking technologies in oral pharmaceuticals: recent developments and approaches. Drug Dev Ind Pharm 2004;30:429-48.

9. Patel NA, Makwana ST, Patel ZP, Solanki SM, Patel MB. Formulation and evaluation of once daily sustained release matrix tablet of pramipexole dihydrochloride. Int J Pharm Res Scholars 2012;1:370-6

10. Ain S, Kumar B, Pathak K. Development and characterization of controlled release famotidine matrix tablets containing complexes. Int J Appl Pharm 2017;9:38-46.

11. Rajendra Prasad A, Ratna JV. Development and validation of a simple UV-spectrophotometric method for the determination of ciprofloxacin HCL present in taste masked drug resin complex. Int J Appl Pharm 2018;10:37-41.

12. Sanghavi NM. Ion-exchange resins as matrix for controlled drug release. Indian Drugs 1988;26:27-32.

13. Prajapati BG, Patel SN. Formulation, evaluation, and optimization of the orally disintegrating tablet of cinnarizine. E-J Sci Technol 2010;1:9-21.

14. Patel BP, Patel JK, Rajput GC, Thakor RS. Formulation and evaluation of mouth dissolving tablets of cinnarizine. Indian J Pharm Sci 2010;72:522-5.

15. Yadav K, Yadav D, Srivastava A. Evaluation of hydrophilic, hydrophobic and waxy matrix excipients for sustained release tablets of venlafaxine hydrochloride. Drug Dev Ind Pharm 2013;39:1197-206.

16. Khar RK, Mohapatra S, Barik BB. Design and characterization of controlled release matrix tablets of Zidovudine. Asian J Pharm Clin Res 2009;2:54-61.

17. Madgulkar AR. Formulation and optimization of sustained release tablets of venlafaxine resinates using response surface methodology. Indian J Pharm Sci 2009;71:387-94.

18. Lakade $\mathrm{SH}$, Bhalekar MR. Formulation and evaluation of sustained release matrix tablet of anti-anginal drug influence of combination of hydrophobic and hydrophilic matrix former. Res J Pharm Technol 2008;1:410-4.

19. Puttewar TY. Formulation and evaluation of orodispersible tablet of taste masked doxylamine succinate using ion exchange resin. J King Saud Univ 2010;22:229-40.

20. Anand V, Kandrapu R, Garg S. Preparation and evaluation of taste-masked orally disintegrating tablets of prednisolone. Asian J Pharm Sci 2007;6:227-38.

21. Das S, Shrikant L. Taste masking of ciprofloxacin by ion exchange resin. IJPPR 2010;1:7-13.

22. Bolton S. Optimization techniques in pharmaceutical statistics. Practical and Clinical Applications. $3^{\text {rd }}$ ed. New York: Marcel Dekker; 1997.

23. Anthony NA. Pharmaceutical experimental design and interpretation. $2^{\text {nd }}$ ed. Taylor and Francis Group; 2006.

24. Lionberger RA, Lee SL, Lee L. Quality by design: concepts for ANDAs. Adv Appl Pharm Sci J 2008;10:268-76.

25. Guideline IH. Stability testing guidelines: stability testing of new drug substances and products. ICH Q1A (R2) (CPMP/ICH/2736/99); 1999.

26. Singh S. Stability testing during product development. In: Jain NK. Pharmaceutical product development. CBS publisher and distributors India; 2000. p. 272-93. 
27. Bajaj S, Singhla D, Sakhuja N. Stability testing of pharmaceutical products. J Appl Pharm Sci 2012;2 Suppl 3:129-38.

28. Parfati N, Rani KC, Charles N, Geovanny V. Preparation and evaluation of atenolol- $\hat{I}^{2}$-cyclodextrin orally disintegrating tablets using co-process crospovidone-sodium starch glycolate. Int J Appl Pharm 2018;10:190-4.

29. Nagar P, Singh K, Chauhan I, Madhu V, Yasir M, Khan A, et al. Orally disintegrating tablets: formulation, preparation, techniques, and evaluation. J Appl Pharm Sci 2011:4:35-45.

30. Gattu J, Lakshmi P. Comparative evaluation of natural and synthetic superdisintegrants with newer superdisintegrant kyron T-314. Acta Pharm Sci 2011;53:35-44.

31. Prajapati BG, Patel SN. Formulation, evaluation, and optimization of the orally disintegrating tablet of cinnarizine. E-J Sci Technol 2010;1:9-21.

32. Bhowmik D, Chiranjib B, Chandira RM. Fast dissolving tablet: an overview. J Chem Pharm Res 2009;1:163-77.
33. Khanam N, Alam MI, MD Yusuf Ali, QMAI Siddiqui, A Ur Rahman. A review on optimization of drug delivery system with experimental designs. Int J Appl Pharm 2018;10:7-12.

34. Anthony NA. Pharmaceutical experimental design and interpretation. $2^{\text {nd }}$ ed. Taylor and Francis Group; 2006.

35. Lewis GA, Mathieu D, Phan-Tan-Luu R. Pharmaceutical experimental design. New York: Marcel Dekker; 1999.

36. Montgomery DC. Design and analysis of experiments. $5^{\text {th }}$ ed. New York: Wiley; 2001.

37. Patel KN, Mehta TA. Design and optimization of nicardipine hydrochloride push-pull osmotic pump tablet using 32 full factorial design. Int J Pharm Biomed Res 2013;4:155-63.

38. Othiya OM, Patel BA, Patel KN, Patel MM. Formulation and characterization of sustained release matrix tablets of ivabradine using $3^{2}$ full factorial design. Int J Appl Pharm 2018;10:59-66. 